\title{
L'emploi d'une préparation de lipase prégastrique dans la fabrication du fromage Gouda
}

\author{
par \\ R. DELBEKE* ${ }^{*}$ et R. VANDERPOORTEN** \\ Ministère de l'Agriculture, Administration de la Recherche Agronomique, \\ Centre de Recherches Agronomiques de l'Etat, Gand
}

\section{Introduction}

L'accélération du processus de maturation a une grande importance économique, mais ne peut être réalisée au détriment de la qualité du fromage.

On admet généralement que ce sont surtout les acides gras libres inférieurs et, à un moindre degré, les produits de la protéolyse - dont en premier lieu les acides aminés - qui contribuent à la formation du goût du fromage. On peut abréger considérablement le processus de maturation si on parvient à accélérer l'hydrolyse de la matière grasse et la protéolyse tout en maintenant un équilibre favorable entre les composants du goût.

On peut, pour accélérer la protéolyse dans un sens favorable, veiller à introduire dans le fromage jeune un grand nombre de streptocoques lactiques. On peut aussi employer pour la fabrication des souches très actives à pouvoir protéolytique élevé [22, 23]. L'hydrolyse de la matière grasse peut être accrue par l'emploi, dans la fabrication, de préparations de lipase ou de cultures de microorganismes lipolytiques.

La pasteurisation ordinaire (de 15 à $20 \mathrm{~s}$, de $73^{\circ} \mathrm{C}$ à $76^{\circ} \mathrm{C}$ ) détruit, en effet, une grande partie des lipases du lait $[9,25]$. Il en va de même d'une grande partie des enzymes lipolytiques microbiens $[25,17]$, quoique certains soient extrêmement résistants à la chaleur [17]. L'hydrolyse de la matière grasse ne progresse que lentement et reste toujours minime dans le fromage Gouda fabriqué par la méthode normale à partir d'un lait pasteurisé qui n'a pas subi de vaste contamination ultérieure par bactéries lipolytiques. Des chercheurs belges [14] ont constaté, sur un total de 81 fabrications de

\footnotetext{
* Groupe de travail pour l'amélioration de la qualité du fromage belge.

** Station Laitière de l'Etat, Melle.
} 
fromage Gouda $48+$ de $10 \mathrm{~kg}$ et après 14 semaines de maturation, que la matière grasse du fromage présentait un degré d'acidité moyen (nombre de $\mathrm{ml} \mathrm{NaOH} \mathrm{N} / 10$ nécessaire pour neutraliser $10 \mathrm{~g}$ de graisse) de 2,70 seulement, les valeurs extrêmes étant 4,48 et 1,64.

D'autres chercheurs [21] ont trouvé, au cours de certains essais, déjà après 10 et après 20 semaines des degrés d'acidité respectifs de 5,00 et 7,30 dans la matière grasse de fromage fabriqué de lait cru. Les valeurs correspondantes, relevées après le même laps de temps pour la matière grasse de fromages fabriqués du même lait, mais pasteurisé, étaient respectivement de 2,2 et de 3,9 . Les principaux enzymes lipolytiques qui peuvent jouer un rôle important dans l'hydrolyse de la matière grasse du fromage, proviennent de bactéries du genre Pseudomonas et Serratia et des achromobactériacées [21, 24]. Des cultures appropriées de ces groupes de bactéries peuvent donc éventuellement être utilisées en fromagerie.

On est déjà parvenu à réaliser un concentré de lipases du lait $[1,2]$, mais ce genre de préparation ne se trouve pas encore dans le commerce. Si l'on considère la complexité du processus de préparation et le coût de la matière première, il semble douteux que l'on puisse mettre dans le commerce dans un avenir plus ou moins proche et à un prix économiquement justifié des lipases de lait destinées à la fromagerie.

On peut toutefois se procurer des préparations de lipases d'autres provenances. Quelques-unes sont d'origine végétale, beaucoup d'origine animale. La plupart ont une action spécifique et l'on sait, par exemple, que les préparations de lipase extraite de pancréas ne libèrent que peu d'acides gras volatils mais, par contre, de grandes quantités d'acides gras supérieurs [7, 11].

Une firme américaine offre des préparations de lipase extraite de glandes prégastriques animales et qui libèrent des quantités relativement importantes d'acides gras volatils de la matière grasse butyrique. Ces préparations s'emploient surtout aux Etats-Unis dans la fabrication de fromages de types italiens. Quelques fabrications parallèles de fromage Gouda $48^{+}$de $10 \mathrm{~kg}$ ont été entreprises avec une préparation de ce genre.

\section{Description de l'expérience}

\section{1) La préparation de lipase}

La Marschall Dairy Laboratory, Inc. vend des préparations dénommées Marschall Lipase Powders no 300, 400 et 600. D'après les données communiquées par le fabricant [13], ces poudres contiennent des lipases prégastriques animales extraites de glandes comestibles de la bouche et de la gorge. La poudre no 300 contient exclusivement des lipases provenant de chevreaux, la $n^{\circ} 400$ est un mélange de lipases extraites de glandes de chevreaux et d'agneaux et la $n^{\circ} 600$ 
est préparée exclusivement de glandes prélevées sur des veaux, Les seuls produits intervenant dans la fabrication et la standardisation des poudres sont le sel, la poiddre de lait écrémé et le lactose. La dispersion des poudres et leur dissolution partielle dans l'eau s'obtiennent facilement.

Les trois types de poudre sont surtout employés aux Etats-Unis dans la fabrication de fromages de goût plus ou moins piquant, principalement de fromages de types italiens. Les fromages italiens étaient fabriqués jadis à l'aide d'une pâte de présure obtenue en broyant des estomacs d'animaux pas encore sevrés. En plus de présure, ces pâtes contiennent du lait caillé et donc, inévitablement, des lipases. Mais elles contiennent aussi toujours de nombreuses bactéries indésirables et c'est pourquoi beaucoup d'entreprises modernes leur ont substitué des extraits de présure et des préparations de lipases exempts de germes.

Le fabricant [13] recommande la poudre $n^{\circ} 300$ quand on veut obtenir un goût très piquant. La poudre $n^{\circ} 600$ est utilisée pour les fromages de goût moins prononcé. Cette préparation no 600 est d'ailleurs beaucoup utilisée dans l'industrie des denrées alimentaires pour conférer une saveur généreuse et spécifique à des produits comprenant de la graisse butyrique. Le fromage, le beurre, la crème de lait, le lait évaporé, la poudre de lait entier et même le lait frais sont autant de substrats favorables à la formation de ce goût spécifique et peuvent ensuite servir à la fabrication de produits de consommation tels que les plats, sauces et potages au fromage, les produits chocolatés, les jus, les pâtes (macaroni, nouilles...), etc.

Toujours selon le fabricant [13], chacune des trois poudres est en mesure, par lipolyse de la graisse butyrique, de libérer des acides gras dans des proportions reproductibles, de manière à procurer un goût spécifique. Ces proportions sont reproduites au tableau 1. Le fabricant signale que la standardisation des poudres est réalisée selon la méthode élaborée par Ramsey [19] pour la détermination du pouvoir enzymatique.

Ce tableau montre clairement que la poudre $n^{\circ} 600$ libère, en pourcentage, nettement moins d'acide butyrique. C'est pourquoi nous l'avons choisie pour nos fabrications parallèles.

D'après le fabricant [13], l'action enzymatique dans un substrat de graisse butyrique est optimale à un $\mathrm{pH}$ de 6,2 et une température de $28^{\circ} \mathrm{C}$ à $37^{\circ} \mathrm{C}$. Elle dépend aussi de l'état physique de la graisse et est optimale quand celle-ci est bien émulsionnée. L'homogénéisation est aussi favorable à l'activité enzymatique parce qu'elle augmente la surface totale des globules de graisse.

Il va de soi que la composition du fromage et les facteurs de maturation extérieurs peuvent modifier les proportions spécifiques d'acides gras libérés indiquées au tableau 1. 
La pasteurisation inactive totalement les préparations. En solution aqueuse, l'inactivation commence même à $49^{\circ} \mathrm{C}$ à se produire relativement vite.

Le fabricant [13] recommande de conserver les lipases à une température inférieure à $0^{\circ} \mathrm{C}$. Déjà 10 p. 100 de l'activité enzymatique se perd en l'espace d'un an quand la préparation est gardée par $-7^{\circ} \mathrm{C}$. La perte d'activité atteint $35 \mathrm{p} .100$ par $10^{\circ} \mathrm{C}$.

Le prix actuel des poudres peut être estimé à environ $950 \mathrm{FB}$ le $\mathrm{kg}$ pour le $\mathrm{n}^{\circ} 300$, à $850 \mathrm{FB}$ pour le $\mathrm{n}^{\circ} 400$ et à $500 \mathrm{FB}$ pour le $\mathrm{n}^{\circ} 600$. Les prix à l'achat de grandes quantités (100 kg et plus) sont moins élevés.

\section{2) Essais et schéma d'analyse}

Le fabricant [13] conseille d'employer de 3 à $13 \mathrm{~g}$ de poudre de lipase par $100 \mathrm{l}$ de lait quand on veut fabriquer des fromages de types italiens de goût plus ou moins piquant. La lipolyse libérant de grandes quantités d'acide butyrique, nous avons préféré utiliser des doses moins élevées pour nos fabrications de fromage Gouda $48^{+}$ de $10 \mathrm{~kg}$.

\section{TABLEAU 1}

Proportions (p. 100 mol.) d'acides gras formés dans une pâte de graisse butyrique. Données fournies par le fabricant

\begin{tabular}{l|r|r|r}
\hline \multicolumn{1}{c|}{ Acides gras } & $\mathrm{N}^{\circ} 300$ & $\mathrm{~N}^{\circ} 400$ & $\mathrm{~N}^{\circ} 600$ \\
\cline { 2 - 3 } & & & \\
\hline Acide butyrique & 45,9 & 42,5 & 36,3 \\
Acide capronique & 18,2 & 16,0 & 11,0 \\
Acide caprylique & 5,0 & 5,3 & 5,1 \\
$\begin{array}{l}\text { Acide caprique } \\
\text { Acide laurique et acides gras } \\
\text { supérieurs }\end{array}$ & 7,8 & 7,1 & 5,9 \\
& 23,2 & 29,3 & 41,6 \\
\hline
\end{tabular}

La Marschall Lipase Powder $\mathrm{n}^{\circ} 600$ a été employée pour cinq fabrications parallèles, à des doses respectives de $0,5,1,1,5,2$ et $3 \mathrm{~g}$ par 1001 de lait.

Dans les essais avec des doses de 0,5 et $1 \mathrm{~g}$, le lait reçu le matin a été immédiatement transformé en fromage. Dans les trois autres essais, le lait a été gardé cru à $5^{\circ} \mathrm{C}$ pendant $24 \mathrm{~h}$ avant d'être traité. Les températures et durées de pasteurisation respectives ont été de $73^{\circ} \mathrm{C}$ à $75^{\circ} \mathrm{C}$ et de 15 à $20 \mathrm{~s}$.

Le dépistage d'éventuelles substances inhibitrices a été effectué et le titre d'acidité, le $\mathrm{pH}$, le nombre de germes et le nombre de bactéries lipolytiques ont été déterminés, aussi bien pour le lait cru 
que pour le lait pasteurisé. L'activité du ferment lactique a été contrôlée.

Pour toutes les fabrications nous avons utilisé $20 \mathrm{~g}$ de salpêtre, $28 \mathrm{ml}$ de présure (force $1 / 10000$ ) et $0,7 \mathrm{l}$ de ferment lactique par 1001 de lait. La quantité requise de poudre de lipase a été versée, $15 \mathrm{mn}$ avant l'emploi, dans 20 fois son volume d'eau froide; cette solution a été remuée régulièrement et ajoutée au lait quelques minutes avant l'emprésurage de celui-ci.

Les deux fabrications de chaque essai parallèle ont été conduites d'après les schémas chronologiques et de température identiques. Les différences d'un essai à l'autre ont également été minimes. La durée et la température moyennes de coagulation ont été de $33 \mathrm{mn}$ et de $30,6^{\circ} \mathrm{C}$. Les valeurs correspondantes du brassage à sec ont été de $49 \mathrm{mn}$ et de $34^{\circ} \mathrm{C}$.

Les teneurs en eau, en matière grasse et en sel des fromages ont été déterminées après 4 semaines. Le nombre de bactéries lipolytiques, le $\mathrm{pH}$, le degré d'acidité de la matière grasse et la quantité d'acides gras libres volatils ont été déterminés à $2,4,6,10,14$ et 20 semaines.

Le goût des fromages a été apprécié aux mêmes moments par 9 experts selon le test triangulaire [3].

Toutes les déterminations physico-chimiques ont été effectuées sur des échantillons découpés en secteurs (1/8 du fromage) et les déterminations bactériologiques sur des échantillons prélevés à la sonde.

\section{3) Méthodes d'analyse}

La présence de substances inhibitrices dans le lait a été recherchée par l'épreuve du yaourt [16].

Le nombre de germes a été déterminé sur Plate Count Agar ( $3 \mathrm{j}$ d'incubation à $30^{\circ} \mathrm{C}$ ) et le nombre de bactéries lipolytiques sur Tributyrin Agar ( $5 \mathrm{j}$ d'incubation à $25^{\circ} \mathrm{C}$ ). Une suspension de fromage a été préparée à l'aide d'une solution à 2 p. 100 de citrate de sodium. Selon certains auteurs [6], le nombre de bactéries capables d'hydrolyser la tributyrine sans dégrader la graisse du beurre ne serait que minime et plus petit que l'on n'admettait naguère.

L'épreuve d'activité du ferment lactique a été effectuée comme exposé sous les références bibliographiques [23, 15].

La teneur en extrait sec a été déterminée par dessiccation à $105^{\circ} \mathrm{C}$, la teneur en matière grasse par la méthode de Van Gulik [4], la teneur en sel conformément à la norme éditée par la Fédération Internationale de Laiterie [5] et le $\mathrm{pH}$ par la méthode décrite sous la référence [20].

Le degré d'acidité de la matière grasse du fromage, dont il est question ici, est le nombre de $\mathrm{ml} \mathrm{NaOH} \mathrm{N} / 10$ nécessaire pour neu- 
traliser $10 \mathrm{~g}$ de cette matière grasse. Il est un indice valable de la quantité d'acides gras inférieurs [20], dont le rôle dans la formation du goût du fromage est précisément si important.

Différentes méthodes ont déjà été utilisées pour isoler du fromage les produits de dégradation de sa matière grasse sans qu'ils soient pollués par d'autres acides. Les acides gras sont tous bien solubles dans la matière grasse qui lorsqu'on l'isole, en retient la plus grande partie en solution. Au cours de certaines fermentations, des acides gras inférieurs peuvent être produits à partir de substances non grasses (les acides formique et acétique à partir du lactose, les acides propionique et butyrique à partir de lactates). Il semble bien que lorsque la matière grasse de l'échantillon en est isolée par pressage avant la détermination des acides gras libres solubles dans cette matière grasse, il n'y a guère de risques que de grandes quantités d'acides gras provenant du lactose et du lactate soient englobées dans la détermination. Certains auteurs [18] ont appliqué la méthode d'extraction du jus par pressage et ont démontré que la perte d'acides gras y est plus faible que dans d'autres méthodes. Nous renvoyons au [14] de la bibliographie pour une description détaillée de cette méthode.

Les acides gras volatils libres du fromage ont été déterminés par chromatographie en phase gazeuse. Après addition de $\mathrm{H}_{2} \mathrm{SO}_{4}$, l'échantillon de fromage a été soumis à une distillation à la vapeur. Les acides gras volatils du distillat ont ensuite été neutralisés et le distillat évaporé. Les acides gras ont été finalement libérés de leurs savons par hydrolyse et recueillis dans de l'hexane, dont 1,5 à $3 \mu$ l ont été injectés dans un chromatographe à phase gazeuse. Celui-ci était un F and M 180 Series Research Chromatograph avec un détecteur à ionisation de flamme, une colonne à base d'acide sébacique à 20 p. 100 en guise de phase stationnaire et un support de chromosorb WAW $60 / 80$ mesh. La température de la colonne était de $130^{\circ} \mathrm{C}$ et le débit de gaz porteur (azote) de $60 \mathrm{ml} / \mathrm{mn}$. L'acide heptanoïque était utilisé en standard interne. En procédant par la méthode décrite, on détermine aussi, par la même opération, les sels des acides gras volatils libres.

\section{Résultats et discussions}

\section{1) Lait cru, lait de fromagerie et ferment lactique}

Le lait cru ne contenait pas de substances inhibitrices. Le titre d'acidité et la valeur pH moyens étaient de 17,50 Dornic et 6,7.

Le lait de fromagerie a été échantillonné dans la cuve. Le nombre moyen de germes était de 150000 et celui des bactéries lipolytiques de 9000 .

La hausse moyenne du titre d'acidité du lait de fromagerie pendant l'épreuve d'activité a atteint 33० Dornic. 


\section{2) Données sur la composition du fromage}

Les valeurs moyennes relatives à l'évolution du $\mathrm{pH}$ et aux teneurs en eau, en sel et en matière grasse des fromages à 4 semaines sont rassemblées dans le tableau 2 .

Les écarts en composition entre les fromages témoins et les fromages à la lipase sont donc plutôt minimes, de sorte que les différences relevées sous le rapport de la lipolyse et du goût peuvent bel et bien être imputées aux quantités de préparation de lipase ajoutées.

\section{$T A B L E A U 2$}

Résultats moyens de l'analyse des fromages à la lipase et des fromages témoins

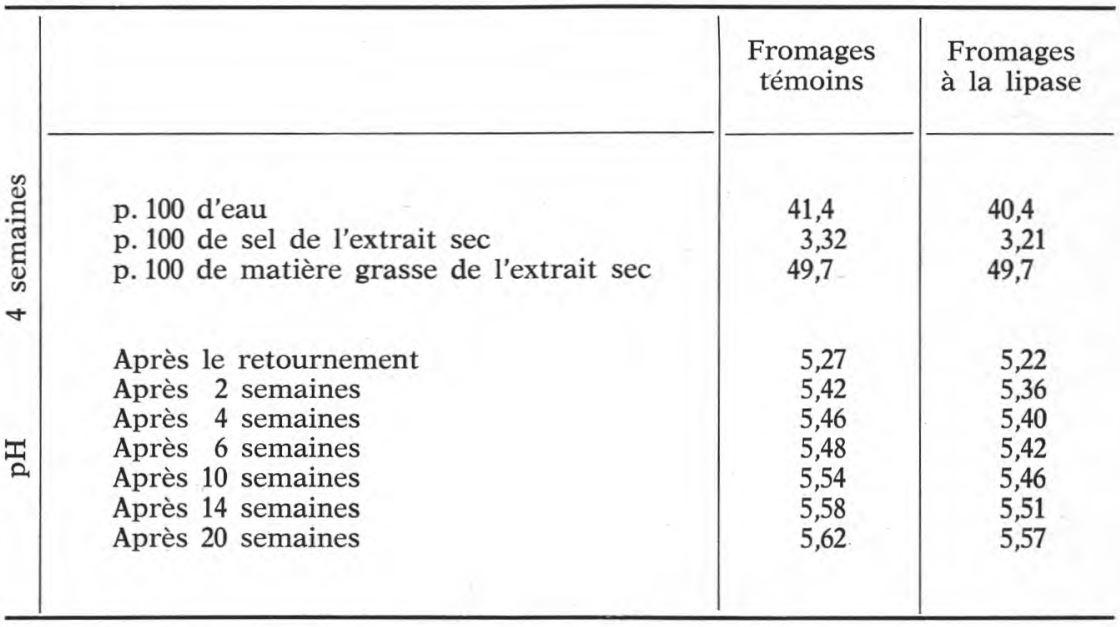

TABLEAU 3

Nombres moyens, en milliers par g, de bactéries lipolytiques du fromage, relevés à différents stades

\begin{tabular}{l|c}
\hline & $\begin{array}{c}\text { Nombres moyens de bactéries } \\
\text { lipolytiques en milliers par g }\end{array}$ \\
\cline { 2 - 2 } Fromage après le pressage & 203 \\
Fromage après le retournement & 129 \\
Fromage à 2 semaines & 77 \\
Fromage à 4 semaines & 21 \\
Fromage à 6 semaines & 11 \\
Fromage à 10 semaines & 6 \\
Fromage à 14 semaines & 5 \\
Fromage à 20 semaines & 1 \\
\end{tabular}




\section{3) Nombre de bactéries lipolytiques}

Le tableau 3 permet de suivre l'évolution du nombre de bactéries lipolytiques du fromage.

Les fromages témoins et les fromages à la lipase de chaque essai parallèle contenaient aux différents stades des nombres à peu près égaux de bactéries lipolytiques.

Le nombre moyen relativement élevé trouvé dans le fromage après le pressage est principalement imputable à la valeur trouvée pour un seul essai.

Après le retournement, ce nombre avait déjà baissé de près de la moitié de ce qu'il était après le pressage. Il ne s'élevait plus qu'à $21000 \mathrm{au}$ g après 4 semaines et qu'à 1000 au g après 20 semaines. Un chercheur néerlandais [21] a constaté une extinction parallèle des bactéries lipolytiques au cours de ses propres expériences.

4) Degré d'acidité de la matière grasse du fromage

Le tableau 4 reproduit les degrés d'acidité moyens, maximaux et minimaux de la matière grasse des fromages témoins et des fromages à la lipase.

\section{TABLEAU 4}

Degré d'acidité de la matière grasse à différents stades de maturation du fromage

\begin{tabular}{|c|c|c|c|c|c|c|c|c|}
\hline \multirow{3}{*}{ 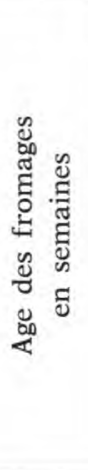 } & \multicolumn{8}{|c|}{ Degré d'acidité de la matière grasse } \\
\hline & \multicolumn{3}{|c|}{ Fromages témoins } & \multicolumn{5}{|c|}{ Fromages à la lipase } \\
\hline & 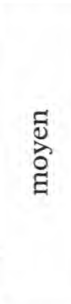 & 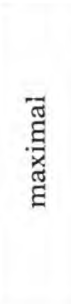 & $\stackrel{\widetilde{\Xi}}{\stackrel{\Xi}{\Xi}}$ & 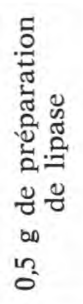 & 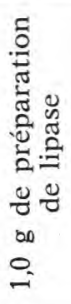 & 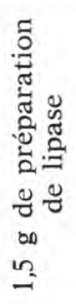 & 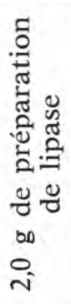 & 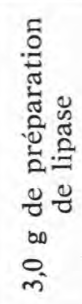 \\
\hline 2 & 1,65 & 2,16 & 1,30 & 2,10 & 2,78 & 3,82 & 4,21 & 4,15 \\
\hline 4 & 1,78 & 2,27 & 1,26 & 2,50 & 3,70 & 4,09 & 4,86 & 5,45 \\
\hline 6 & 2,03 & 2,37 & 1,54 & 2,61 & 3,86 & 4,67 & 5,66 & 7,86 \\
\hline 10 & 2,63 & 2,99 & 2,36 & 4,31 & 6,04 & 7,96 & 7,98 & 9,18 \\
\hline 14 & 3,06 & 3,41 & 2,55 & 4,82 & 6,26 & 8,12 & 8,06 & 10,28 \\
\hline 20 & 3,46 & 4,38 & 2,94 & 5,79 & 7,46 & 8,40 & 8,78 & 12,67 \\
\hline
\end{tabular}


Le degré d'acidité moyen des fromages témoins était très proche de la valeur moyenne relevée, pour les fromages de 81 fabrications, lors d'une étude antérieure de la fabrication du Gouda [14] : une moyenne de 2,70 après 14 semaines, avec un maximum de 4,48 et un minimum de 1,64 .

Le degré d'acidité des fromages fabriqués à l'aide de $0,5 \mathrm{~g}$ de poudre de lipase par $100 \mathrm{l}$ de lait était, à 2 semaines, déjà nettement supérieur à la moyenne relevée pour les fromages témoins. Pour une dose aussi faible de poudre de lipase, le degré d'acidité à 20 semaines $(5,79)$ était presque le double de la moyenne relevée pour les fromages témoins $(3,46)$.

Avec 1,5 g de poudre de lipase, le degré d'acidité a atteint très rapidement des valeurs relativement élevées : 4,67 après 6 semaines, mais déjà 7,96 à 10 et 8,40 à 20 semaines.

L'analyse chromatographique en phase gazeuse a démontré que la maturation des fromages à la lipase libérait surtout de grandes quantités d'acide butyrique. Il est indispensable de tenir compte de ceci dans l'interprétation des données fournies pour le degré d'acidité de fromages à la lipase.

Comme dit plus haut, la matière grasse du fromage en a été extraite par pressage et l'acide butyrique se révèle être le seul des acides gras volatils inférieurs susceptible de passer à la phase aqueuse en quantités relativement considérables [18]. Les valeurs indiquées au tableau 4 pour les fromages à la lipase sont donc probablement un peu inférieures à la réalité.

Signalons encore qu'aucun rapport significatif n'a pu être démontré entre le nombre de bactéries lipolytiques et le degré d'acidité de la matière grasse des fromages témoins.

\section{5) Acides butyrique, capronique et caprylique}

Le tableau 5 donne, en $\mathrm{mg}$ par $\mathrm{kg}$ de fromage, les quantités d'acides butyrique, capronique et caprylique déterminées à différents stades.

La bibliographie ne comportant aucune analyse complète des acides gras libres du fromage Gouda, des chercheurs américains [10] ont analysé 4 échantillons de Gouda américain (acquis sur différents marchés locaux). Les valeurs moyennes, maximales et minimales qu'ils ont trouvées pour les trois acides gras précités ont également été reprises dans ce tableau. Les auteurs américains n'indiquent pas l'âge des fromages qu'ils ont analysés, mais il s'agissait très probablement de fromages en âge d'être consommé.

Ces auteurs ne font pas mention des résultats d'analyse des cinq fromages dont il est question dans la référence [11]. Ces dernières données indiquent toutefois en p. $100 \mathrm{~mol}$ les quantités d'acides gras libres trouvées dans la matière grasse du fromage. 
TABLEAU 5. - Nombre de mg d'acides gras par $\mathrm{kg}$ de fromage

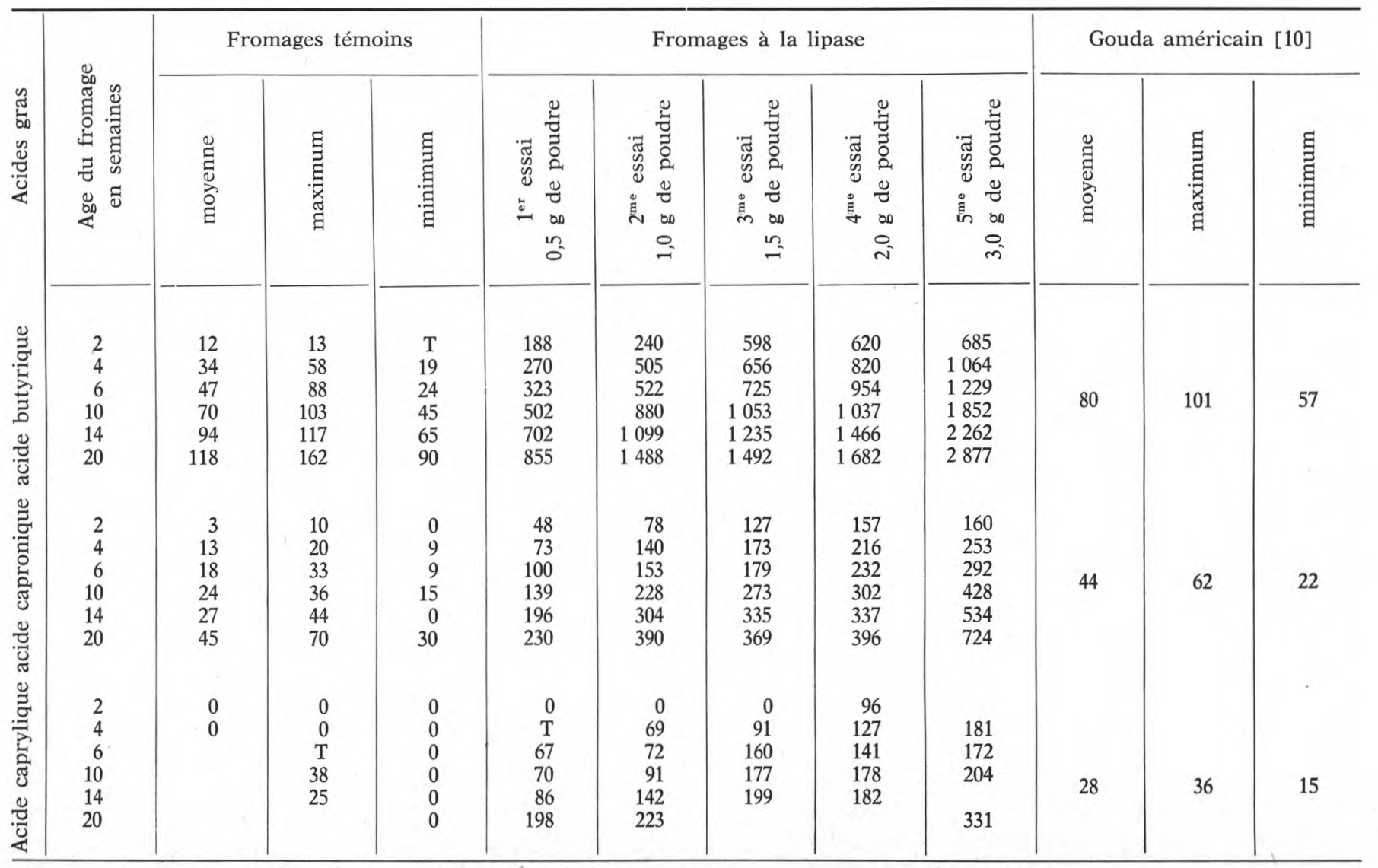

$T=$ traces 
L'acide butyrique était présent en abondance dans les fromages à la lipase et a fortement influencé le goût de ceux-ci. On a même relevé à 2 semaines dans les fromages fabriqués avec seulement $0,5 \mathrm{~g}$ de poudre de lipase par 1001 de lait des valeurs d'acide butyrique et d'acide capronique supérieures aux moyennes des fromages témoins âgés de 20 semaines.

Si l'on tient compte de la différence en poids moléculaire, on peut déduire du tableau 5 que les fromages à la lipase contiennent environ les proportions numériques suivantes de molécules d'acides gras :

- acide butyrique / acide capronique

égale à $7 / 1$

- acide butyrique / acide caprylique

égale à $12 / 1$

- acide capronique / acide caprylique

égale à $1,7 / 1$

Ces proportions sont quelque peu différentes de celles que l'on peut déduire du tableau 1, dont les chiffres - selon le fabricant [13] valent pour la graisse de beurre et pour une lipolyse se déroulant dans des conditions optimales :

- acide butyrique / acide capronique

- acide butyrique / acide caprylique

- acide capronique / acide caprylique

égale à $3,6 / 1$

égale à $7,1 / 1$

égale à $2,2 / 1$

On peut aussi déduire du tableau 5 que les rapports réciproques entre les quantités libérées des trois acides gras pris en considération sont relativement bien reproductibles. Prenons, à titre d'exemple, les fromages fabriqués à l'aide de 1,5 et de 3,0 de poudre de lipase. Pour les rapports acide butyrique/acide capronique, acide butyrique / acide caprylique et acide capronique/acide caprylique, nous trouvons (en chiffres arrondis) les valeurs suivantes après respectivement :

4 semaines : 1,5 $\mathrm{g}$ de lipase : 3,8 - 7 - 1,9.

$3 \mathrm{~g}$ de lipase : 4,2 - $6-1,4$.

6 semaines : $1,5 \mathrm{~g}$ de lipase : 4,1 - 5 - 1,1.

3 g de lipase : 4,2 - $7-1,7$.

10 semaines : $1,5 \mathrm{~g}$ de lipase : 3,9-6 - 1,5.

$3 \mathrm{~g}$ de lipase : $4,3-9-2,1$.

Ces valeurs ne sont donc pas exagérément disparates aux différents stades de maturation et pour des quantités différentes de poudre de lipase. Quant au rapport acide butyrique / acide capronique, l'écart entre les valeurs extrêmes n'est que de $0,5 \quad(4,3$ à 10 semaines et pour $3 \mathrm{~g}$ de poudre et 3,8 à 4 semaines et pour 1,5 $\mathrm{g}$ de poudre). Les rapports acide butyrique / acide caprylique et acide capronique / acide caprylique présentent des écarts quelque peu supérieurs.

L'emploi d'une préparation de lipase du genre en cause permet donc de standardiser à un degré élevé la quantité d'acides gras libérés. Le choix adéquat de la quantité à additionner devrait donc permettre à un moment déterminé l'obtention dans le fromage d'un nombre désiré de mg d'acides gras libres en proportions déterminées. 
TABLEAU 6

Résultats d'analyse des expertises par le test triangulaire pour un risque de première espèce et un niveau $\alpha=0,05$

\begin{tabular}{c|c|c|c|c|c}
\hline $\begin{array}{c}\text { Age des } \\
\text { fromages } \\
\text { en semaines }\end{array}$ & $\begin{array}{c}0,5 \mathrm{~g} \text { de } \\
\text { préparation } \\
\text { de lipase }\end{array}$ & $\begin{array}{c}1 \mathrm{~g} \text { de } \\
\text { préparation } \\
\text { de lipase }\end{array}$ & $\begin{array}{c}1,5 \mathrm{~g} \text { de } \\
\text { préparation } \\
\text { de lipase }\end{array}$ & $\begin{array}{c}2,0 \mathrm{~g} \text { de } \\
\text { préparation } \\
\text { de lipase }\end{array}$ & $\begin{array}{c}3,0 \mathrm{~g} \text { de } \\
\text { préparation } \\
\text { de lipase }\end{array}$ \\
\hline 2 & $\mathrm{D}$ & $\mathrm{D}$ & $\mathrm{L}$ & $\mathrm{L}$ & $\mathrm{L}$ \\
4 & $\mathrm{D}$ & $\mathrm{D}$ & $\mathrm{L}$ & $\mathrm{L}$ & $\mathrm{L}$ \\
6 & $\mathrm{D}$ & $\mathrm{T}$ & $\mathrm{D}$ & $\mathrm{D}$ & $\mathrm{D}$ \\
10 & $\mathrm{~T}$ & $\mathrm{D}$ & $\mathrm{T}$ & $\mathrm{T}$ & $\mathrm{T}$ \\
14 & $\mathrm{~T}$ & $\mathrm{D}$ & $\mathrm{T}$ & $\mathrm{T}$ & $\mathrm{T}$ \\
20 & $\mathrm{~T}$ & $\mathrm{D}$ & $\mathrm{T}$ & $\mathrm{T}$ & $\mathrm{T}$ \\
\hline
\end{tabular}

$\mathrm{D}=$ les chances de conclure erronément à une différence de goût ne sont que de 5 sur 100.

$\mathrm{L}=$ les chances d'accorder à tort la préférence aux fromages à la lipase ne sont que de 5 sur 100 .

$\mathrm{T}=$ les chances d'accorder à tort la préférence aux fromages témoins ne sont que de 5 sur 100.

\section{6) Expertise}

a) Texture : La formation d'yeux a été expertisée en même temps que le goût. Le total des résultats d'expertise a fait apparaître que les différences entre les fromages d'une même fabrication étaient certainement aussi grandes que les différences constatées entre les fromages à la lipase et les fromages témoins d'un même essai parallèle.

On peut donc admettre que l'emploi d'une préparation de lipase, en doses allant jusqu'à $3 \mathrm{~g}$ par $100 \mathrm{l}$ de lait, n'influe nullement ou seulement qu'à un degré minime sur la texture du fromage.

b) Goût : Neuf experts ont jugé le goût à différents stades et par le test triangulaire [3]. Ceci a permis d'exploiter les résultats d'expertise pour un risque choisi de première espèce [8]. Le tableau 6 donne l'analyse des résultats obtenus par le test triangulaire, donnés pour un niveau $\alpha=0,05$.

Il ressort de ce tableau que la plupart des experts a toujours décelé une différence de goût. Ce fut, entre autres, déjà le cas après 
2 semaines pour l'essai où l'on n'avait employé que $0,5 \mathrm{~g}$ de poudre par 1001 de lait.

Là où on avait employé de 1,5 à $3 \mathrm{~g}$ de poudre, les experts ont accordé, à 2 et à 4 semaines, la préférence aux fromages à la lipase, alors qu'ils n'ont pris aucune décision pour les fabrications à 0,5 et à $1 \mathrm{~g}$.

A partir de la $10^{\text {me }}$ semaine, la préférence s'est portée sur les fromages témoins pour 4 des 5 doses utilisées. Les experts ont bien distingué une différence de goût pour l'essai à $1 \mathrm{~g}$ de préparation, mais n'ont pas été unanimes quant à leurs préférences. Il y a là une certaine contradiction avec le fait que les experts ont, à partir de la $10^{\mathrm{me}}$ semaine, donné la préférence aux fromages témoins, même quand les fromages à la lipase n'avaient été fabriqués qu'avec $0,5 \mathrm{~g}$ de poudre. Il serait prématuré de tirer une conclusion de cette anomalie.

\section{Conclusions}

1) La préparation de lipase employée dans ces essais est en mesure de libérer en peu de temps d'importantes quantités d'acides gras volatils de la matière grasse du fromage.

Même si on l'emploie à de très faibles doses $(0,5 \mathrm{~g}$ par $1001 \mathrm{de}$ lait), on obtient déjà après 2 semaines des quantités d'acide butyrique et d'acide capronique nettement plus élevées qu'après 20 semaines dans les fromages témoins.

2) Les proportions réciproques de quantités libérées d'acides butyrique, capronique et caprylique sont relativement bien reproductibles, de sorte qu'il devient possible de les standardiser et d'obtenir aussi, par un choix adéquat de la dose de poudre, à un moment déterminé de la maturation du fromage une quantité déterminée de ces acides gras.

3) Pour des doses de 0,5 à $1 \mathrm{~g}$ de poudre par $100 \mathrm{l}$ de lait, la plupart des experts ont bien décelé des différences de goût entre les fromages à la lipase et les fromages témoins âgés de 2 et de 4 semaines, mais quelques-uns seulement ont accordé leur préférence aux fromages à la lipase. Ils ont été plus nombreux à préférer aux fromages témoins des mêmes âges les fromages fabriqués à l'aide de 1,5 à $3 \mathrm{~g}$ de poudre de lipase.

A partir de la $10^{\text {me }}$ semaine, la préférence est allée pratiquement toujours aux fromages témoins, quelle que fut la quantité de poudre de lipase employée au cours des essais.

Il existe des indices que certains consommateurs de fromage apprécient les fromages à la lipase tant que la dose utilisée n'est pas trop forte (moins de $3 \mathrm{~g}$ ). Cette tendance n'est pas sans intérêt car un type de Gouda au goût moins classique, mais apprécié d'un 
certain nombre de consommateurs, pourrait introduire plus de variété dans la production.

4) Si l'on admet que c'est surtout la grande quantité d'acide butyrique qui, dominant les quantités d'acides capronique et caprylique, influe défavorablement sur le goût après quelques semaines de maturation, la préparation essayée doit être qualifiée moins propre à la fabrication de fromage Gouda.

On peut chercher une solution à ceci dans une combinaison de la poudre en cause avec d'autres produits à la lipase, que l'on peut se procurer dans le commerce. On sait que des préparations de lipase extraites de pancréas libèrent proportionnellement beaucoup moins d'acide butyrique et donnent surtout des acides gras libres supérieurs $[7,11]$.

Une autre possibilité réside dans le recours à des bactéries qui hydrolysent la matière grasse. Certains libèrent relativement peu d'acide butyrique et donnent des rapports plus favorables entre les quantités d'acides butyrique, capronique et caprylique libérées [12,26].

On peut aussi envisager la possibilité de combiner une préparation de lipase avec des souches bactériennes déterminées.

\section{Rés u m é}

L'accélération du processus de maturation a son importance économique, mais ne peut être réalisée au détriment de la qualité du fromage.

Les acides gras volatils libres jouent un rôle très important dans la formation du goût du fromage. On peut accélérer l'hydrolyse de la matière grasse du fromage fabriqué de lait pasteurisé en recourant à des préparations de lipase ou à des cultures de bactéries lipolytiques.

Une préparation de lipase extraite de glandes prégastriques de veaux a été utilisée pour quelques fabrications parallèles (fromage Gouda de $10 \mathrm{~kg}$ ) et à des doses allant de 0,5 à $3 \mathrm{~g}$ par 1001 de lait.

Même avec $0,5 \mathrm{~g}$ de cette préparation par $100 \mathrm{I}$ de lait, le fromage contenait déjà après 2 semaines des quantités considérables d'acides gras volatils libres, dont les rapports réciproques se sont révélés franchement reproductibles, de sorte qu'une standardisation est possible.

Pour obtenir rapidement un goût nettement marqué, il a fallu au moins $1,5 \mathrm{~g}$ de préparation par $100 \mathrm{l}$ de lait, mais, à cette dose, la préférence de la plupart des experts est allée, à partir de la $10^{\text {me }}$ semaine, aux fromages témoins. Certains consommateurs ont toutefois apprécié le goût légèrement différent des fromages fabriqués à la lipase, ce dont on pourrait peut-être tirer profit pour introduire plus de variété dans la production. 


\section{S u m m a ry}

Acceleration of the ripening process is of importance economically but must not be applied to the detriment of the cheese quality.

Free volatile fatty acids are of great importance as regards the development of the flavour. To accelerate the fat hydrolysis in cheese made of pasteurized milk, lipase preparations or cultures of lipolytic micro-organisms may be used.

A number of parallel tests (Gouda cheese, $10 \mathrm{~kg}$ ) have been carried out with a lipase preparation obtained from pregastric glands of calves, in doses ranging from 0.5 to $3 \mathrm{~g}$ per $100 \mathrm{l}$ milk.

Considerable amounts of free volatile fatty acids were found after as little as two weeks in the cheese made with the lowest dose, i.e. $0.5 \mathrm{~g}$ per $1001 \mathrm{milk}$. Their ratio appeared to have quite good reproductibility, permitting standardization.

At least $1.5 \mathrm{~g}$ of the preparation per 1001 milk was needed to obtain quickly a clearly defined flavour in the cheese, but at such a dose most of the experts preferred the control cheeses from the tenth week onwards. Some consumers, however, appeared to appreciate the slightly different flavour of the cheeses made with the lipase preparation. This may be an incentive to introduce more variation into production.

\section{Références bibliographiques}

[1] Chandan (R. C.) and Shahani (K. M.) (1963). - Purification and characterization of milk lipase. I. Purification. J. Dairy Sci., 46, 275.

[2] Chandan (R. C.) and Shahani (K. M.) (1963). - Purification and characterization of milk lipase. II. Characterization of the purified enzyme. J. Dairy Sci. 46,530 .

[3] De Man (J. C.) (1960). - De kunst van het keuren. Misset's Zuivel, 66, 104.

[4] De Vleeschauwer (A.), Hendrickx (H.) en Heyndrickx (G.) (1948). - Onderzoekingsmetoden van zuivelprodukten. N.V. Standaard-Boekhandel, Antwerpen.

[5] Fédération Internationale de Laiterie (1961). - Détermination de la teneur en sel du fromage. F.I.L., rue Ortélius, 10, Bruxelles, FIL-IDF 17.

[6] Fryer (T. F.), Laurence (R. C.), Reiter (B.) (1967), - Methods for isolation and enumeration of lipolytic organisms. J. Dairy Sci., 50, 476.

[7] HarPer (W. J.) (1957), - Lipase systems used in the manufacture of Italian cheese. II. Selective hydrolysis. J. Dairy Sci., 40, 556.

[8] Harvard University Press (1955). - Tables of the cumulative binominal probability distribution. Harvard University Press.

[9] Hetrick (J.H.) and Tracy (P. H.) (1948), - Effect of high-temperature short-time heat treatments on some properties of milk. II. Inactivation of the lipase enzyme. J. Dairy Sci., 31, 881.

[10] Iyer Meena, Richardson (T.), Amundson (C. H.) and Tripp (R. C.) (1967). Major free fatty acids in Gouda cheese. J. Dairy Sci., 50, 385. 
[11] Jack (E. L.), Freeman (C. P.), Smith (L. M.) and Mickle (J. B.) (1963). Pancreatic lipase hydrolysis of cow milk fat. J. Dairy Sci., 46, 284.

[12] Khan (I. M.), Chandan (R. C.), Dill (C. W.) and Shahani (K. M.) (1966). Hydrolysis of milk fat by microbial lipases. J. Dairy Sci., 49, 700.

[13] Marschall Dairy Laboratory, Inc. Lipase powders $\mathrm{n}^{\circ} 300,400$ and 600. Marschall Dairy Laboratory, Inc., 14, Proudfit st., Madison 1, Wisconsin (U.S.A.), Spec. sheet $\mathrm{n}^{\circ} 101$.

[14] Naudts (M.), Delbeke (R.) en Waes (G.) (1965). - De bereiding van Goudse kaas. Deel VII. De kaasontleding en de kaaskeuring. Werkgroep ter verbetering van de kwaliteit van de Belgische kaas, Rijkszuivelstation, Melle, 13' Mededeling Werkgroep.

[15] Naudts (M.), Delbeke (R.), Waes (G.) en Hendrickx (H.) (1964). - De bereiding van Goudse kaas. Deel III. Het onderzoek van het kaaszuursel. Werkgroep ter verbetering van de kwaliteit van de Belgische kaas, Rijkszuivelstation, Melle, $8^{\mathrm{c}}$ Mededeling Werkgroep.

[16] Naudts (M.) en WaEs (G.) (1963). - Een eenvoudige proef voor het opsporen van antibiotica in melk. Rijkszuivelstation, Melle.

[17] Pinheiro (A. J. R.), Liska (B. J.) and Parmelee (C. E.) (1965). - Heat stability of lipases of selected psychrophilic bacteria in milk and purdue swisstype cheese. J. Dairy Sci., 48, 983.

[18] RaAdsveld (C. W.) and Mulder (H.) (1949). - The influence of the temperature on the ripening of Edam cheese. Ned. Melk- en Zuiveltijdschr., $3,117$.

[19] Ramsey (H. A.) (1959). - Photometric procedure for determining esterase activity. Clin. Chem., 3, 185.

[20] Ritter (W.), SAHLI (K. W.) und Schilt (P.) (1961). - Restzucker und pHWert im jungen Käse. Schweitz. Milchztg (Lait. Romand), 87 : Wissenschaftliche Beilage $\mathrm{Nr} 77$.

[21] Stadhouders (J.) (1956). - De hydrolyse van vet bij de kaasrijping in verband met de smaak van kaas. Proefschrift ter verkrijging van de graad van doctor in de landbouwkunde, H. Veenman en Zonen, Wageningen.

[22] Stadhouders (J.) (1960). - De eiwithydrolyse tijdens de leceasryping. De enzymen die het eiwit in kass hydrolyzeren. Ned. Melk en Zuiveltijdschr., $14,83$.

[23] StAdHouders (J.) (1961). - De eiwitafbraak in kaas gedurende de rijping. Enige methoden om de rijping te versnellen. Ned. Melk- en Zuiveltijdschr., $15,151$.

[24] Stadhouders (J.) and Mulder (H.) (1958). - Fat hydrolysis and cheese flavour. II. Micro-organisms involved in the hydrolysis of fat in the interior of the cheese. Ned. Melk- en Zuiveltijdschr., 12, 238.

[25] Stadhouders (J.) and Mulder (H.) (1960). - Fat hydrolysis and cheese flavour. IV. Fat hydrolysis in cheese from pasteurized milk. Ned. Melk- en Zuiveltijdschr., 14, 141.

[26] Wrucox (J.C.) Nelson (W. O.) and Wood (W. A.) (1955). - The selective release of volatile acids from butterfat by microbial lipases. J. Dairy Sci., 38, 775 . 